\title{
La pedagogía praxeológica como componente en el proceso de investigación para la formación ciudadana
}

\section{Mauricio Carvajal}

Corporación Universitaria Minuto de Dios. Colombia.

mauricio.carvajal@uniminuto.edu

\section{Resumen}

Este artículo muestra el aporte que trae el enfoque praxeológico frente al desarrollo de competencias ciudadanas y formación ciudadana, a partir de un mecanismo incipiente e incidente en el quehacer pedagógico, formulado desde la educación superior y desarrollado en las aulas infantiles. Esta es una estrategia pedagógica muy aterrizada al reconocimiento de necesidades sociales de manera no convencional, que invita en doble vía a la inclusión, la innovación y al desarrollo de competencias de tipo social. La metodología de la investigación en curso consiste en involucrar a diversos sectores de la sociedad para la reconstrucción de espacios democratizadose incluyentes, a partir de la reflexión y discusión de una formación ciudadana pensada desde y para la población infantil.

\section{Palabras clave}

Educación civica; competencias ciudadanas; aprendizaje activo; praxis pedagógica; estrategias didácticas (Fuente: Tesauro de la Unesco).

Recepción: 2015-07-01 | Envío a pares: 2016-02-07 | Aceptación por pares: 2016-07-22 | Aprobación: 2016-07-24 DOI: 10.5294/edu.2016.19.3.6

Para citar este artículo / To reference this article / Para citar este artigo

Carvajal, M. (2016). La pedagogía praxeológica como componente en el proceso de investigación para la formación ciudadana. Educ. Educ., 19(3), 416-436. DOI: 10.5294/edu.2016.19.3.6 


\title{
Pedagogical Praxeology as a Component in the Investigation Process for Civic Education
}

\begin{abstract}
This paper presents the contributions of the praxeological approach to the development of civic competences and civic education, based on an incipient and incident mechanism on pedagogical practice, shaped in higher education and developed in children's classrooms. This pedagogical strategy is highly linked to the recognition of social needs in a non-conventional way that encourages the inclusion, innovation and the development of social competences. The methodology of this investigation is to involve different sectors of society for the reconstruction of democratized and inclusion-oriented spaces, based on the reflection and discussion of a civic education thought from and for the child population.
\end{abstract}

\section{Keywords}

Civic Education, Civic Competences, Active Learning, Pedagogical Praxis, Didactic Strategies (Source: Unesco Thesaurus). 


\title{
A pedagogia praxiológica como componente no processo de investigação para a formação cidadã
}

\begin{abstract}
Resumo
Este artigo mostra a contribuição do foco praxiológico para o desenvolvimento de competências e formação cidadãs, a partir de um mecanismo incipiente e incidente na atividade pedagógica, que foi formulado desde o ensino superior e desenvolvido nas aulas infantis. Esta é uma estratégia pedagógica focalizada ao reconhecimento de necessidades sociais de um jeito não convencional, que convida à inclusão, à inovação e ao desenvolvimento de competências de tipo social em via dupla. A metodologia da investigação em curso é envolver diversos setores da sociedade para a reconstrução de espaços democratizados e inclusivos, a partir da reflexão e discussão de uma formação cidadã, pensada desde e para a população infantil.
\end{abstract}

\section{Palavras-chave}

Educação cidadã, competências cidadãs, aprendizagem ativa, práxis pedagógica, estratégias didáticas (Fonte: Tesauro da Unesco). 


\section{Introducción}

Desde las políticas públicas sobre educación infantil en Colombia se han formulado algunos mecanismos que ofrecen una atención integral a niños y niñas desde el primer año de edad hasta su juventud (más conocida como la estrategia de Estado denominada "De cero a siempre", del Ministerio de Educación Nacional - MEN). La etapa fundamental para el desarrollo del ser humano está hasta los seis años de vida según los expertos del tema en crecimiento y desarrollo infantil. Por tanto, los desarrollos y aprendizajes que se adquieren durante este periodo son significativos y pueden ser fortalecidos por una educación inicial de calidad de manera integradora, a través de la implementación de programas y proyectos que propicien en el niño y la niña el arte, la literatura, el juego y la exploración de su medio (Ministerio de Educación Nacional, 2014a, p. 4).

Con lo anterior, se hace necesario formular propuestas innovadoras encaminadas a vincular el trabajo de la escuela junto con la responsabilidad que tienen de manera compartida las madres, los padres de familia y demás individuos que tienen a cargo niños y niñas, promoviendo así un proceso de formación y de educación integral, que reconfigure el desarrollo de las competencias ciudadanas desde la educación inicial. Esta propuesta proviene de la academia, en donde se encuentra inserta la función que han tenido las ciencias sociales, y en especial la educación, pero con un altísimo grado de compromiso social desde los procesos educativos y pedagógicos.

En este artículo de reflexión y de divulgación se valora el tema de la pedagogía social, concebida como una manera de atribuir el compromiso que tienen las ciencias de la educación, no solamente en el proceso de formación de individuos, sino además en la gestión e integración del quehacer pedagógico a partir de las necesidades sociales de una comunidad (Bourdieu, 2003, p. 45). El artículo se estructura en cuatro apartados. En el primero se encuentra el planteamiento de la problemática social que se viene presentando en el contexto educativo colom- biano. En el segundo está la fundamentación conceptual y teórica en lo concerniente a la educación inicial y la pedagogía praxeológica o social. Luego, se muestra el método abordado en una investigación que se lleva a cabo en la Corporación Universitaria Minuto de Dios (Uniminuto), relacionado con el desarrollo pedagógico social para la formación ciudadana desde la educación inicial. Y para terminar, se mencionan algunos de los resultados que ha arrojado por el momento la investigación frente a la labor social de las ciencias de la educación, como ciencia aplicada al servicio para y de la comunidad.

\section{Planteamiento de problema}

Primero se describe la línea y temática de investigación; el planteamiento del problema, que se evidencia en el proceso de formación docente en competencias ciudadanas en Colombia. De igual manera, se trae a colación algunos interrogantes que surgen del planteamiento del problema; y por último, el objetivo de la investigación que parte como la formulación de una posible solución al problema.

\section{Línea y temática de investigación}

La línea de investigación del sistema Uniminuto, a la cual le apunta el estudio es: educación, transformación social e innovación (Uniminuto, 2013, p. 6o). Dentro de este lineamiento se buscan procesos de investigación bajo una perspectiva crítica y creativa para la generación de conocimiento, la innovación y el desarrollo educativo social. Por tanto, los temas que hacen referenciase abordan en esta ocasión son la formación ciudadana y la pedagogía praxeológica o social.

\section{Planteamiento del problema}

La existencia de una necesidad por satisfacer se consolida en dos frentes. Por un lado, se encuentra la falta de estrategias pedagógicas que fomenten la formación ciudadana en el proceso de formación de licenciados. El otro frente es la oportunidad que 
se presenta desde las políticas públicas, como lo expone el Plan Decenal de Educación Nacional 20062016; ' y a nivel local en los diferentes planes de desarrollo de los entes territoriales. Estos documentos promueven desde una de sus dimensiones el reconocimiento de la pertinencia para trabajar en pro de la formación para una ciudad educadora, donde se orienta el trabajo a educar personas para el ejercicio de la ciudadanía, la valoración y la recreación de la cultura y la apropiación crítica de la ciudad.

La falta de estrategias pedagógicas para el fomento y el reconocimiento de diversos espacios del entorno de los niños y las niñas, como espacios significativos para el proceso de formación ciudadana, articulados a la adaptación al medio y su contexto sociocultural es evidente en el quehacer del aula. Por tanto, la ausencia de procesos de formación y cualificación docente de competencias ciudadanas desde temprana edad hace que exista una desarticulación de la estructura de enseñanza y aprendizaje con las necesidades del contexto. Esa preparación requiere de profesionales de las ciencias de la educación que desarrollen las competencias sociales necesarias para lograr impartir diversas prácticas educativas que provoquen en la población infantil este ejercicio de la ciudadanía.

Como tal, se adolece de una estructura curricular motivante que permita la formación de las niñas

$1 \quad$ El Plan establece dos temas acordes y aplicables a la formulación y solución del problema de investigación: el primer tema está relacionado con la Educación en y para la paz, la convivencia y la ciudadanía, en donde se plantea el diseño y la aplicación de políticas públicas articuladas que garanticen una educación en y para la paz, la convivencia y la ciudadanía, bajo el enfoque de derechos y deberes, principios de equidad e inclusión social. El segundo tema es el de la Renovación pedagógica y uso de las TIC en la educación, en donde las propuestas tienden al mejoramiento de la infraestructura de las instituciones educativas; a fortalecer la transversalidad curricular en el uso de las TIC y los procesos lectores y escritores; a avanzar en la formación inicial y permanente de docentes y directivos para que centren su labor de enseñanza del estudiante como sujeto activo; a implementar estrategias didácticas activas que faciliten el aprendizaje autónomo, colaborativo y el pensamiento crítico y creativo, entre otros. y los niños. Para corroborar lo anterior, se analizaron los resultados de 183 estudiantes en la evaluación estatal Saber-Pro del Instituto Colombiano para el Fomento a la Educación Superior (ICFES) en el año 2013, para acreditar la titulación de la licenciatura en pedagogía infantil (más del $75 \%$ de los estudiantes se encuentran en quintiles 1 y 2 , considerados como los parámetros más bajos en competencias ciudadanas). Las competencias ciudadanas son categorizadas como genéricas por parte del proceso de aprendizaje de los estudiantes de la licenciatura en Uniminuto - Rectoría Valle. Los resultados dentro del primer periodo del 2013 fueron los siguientes: el $24 \%$ de la población que presentó la prueba se ubicó en el quintil I (muy bajo), el $38 \%$ en un quintil II (bajo), el 20\% en el quintil III (regular), el $14 \%$ en quintil IV (bueno) y solo el $1 \%$ en el quintil $\checkmark$ (muy bueno). Para el 2013-2, el $28 \%$ se ubicó en el quintil I (muy bajo), el 23\% en un quintil II (bajo), el $17 \%$ en el quintil III (regular), el $16 \%$ en quintil IV (bueno) y un incremento significativo del $13 \%$ en el quintil $\mathrm{V}$ (muy bueno). Pero a pesar del crecimiento en las pruebas, las cifras no son muy alentadoras en cuanto a la responsabilidad que tiene un profesional de la educación frente al compromiso y conocimiento idóneo para formar a la población infantil en formación ciudadana.

Lo anterior involucra a diversos actores sociales de la sociedad, no solo a la academia, sino también al sector educativo en general, la familia y demás individuos de la sociedad, en donde se debe entrar a reconocer los roles sociales y a establecer compromisos para promulgar la responsabilidad social compartida para la formación del ciudadano. Por otro lado, el desconocimiento de las políticas púbicas dentro del sector educativo; la manera convencional para formar al docente frente a competencias básicas desconociendo los contextos sociales; la poca disponibilidad de recursos económicos o financieros para el desarrollo de material didáctico adaptado a la realidad, son algunas de las causas de la problemática. Y, como consecuencias, se tiene 
el desarrollo de currículos de formación descontextualizados y desarticulados frente a la formación ciudadana (figura 1).

\section{Pregunta problema}

Con lo anterior, se presenta el primer interrogante frente al planteamiento del problema: ¿cómo inciden las estrategias de enseñanza de los profesores en el proceso de formación docente y quehacer pedagógico para el desarrollo de competencias ciudadanas y sociales desde espacios educativos?

Esto hace que se piense en el diseño e implementación de una estrategia pedagógica que desarrolle procesos educativos para fomentar desde la primera infancia temas de construcción de imaginarios de ciudad a fin de replantear la creación de una nueva cultura frente al sentido de pertenencia por el conocimiento y cuidado de la imagen de la ciudad, los cuales se han perdido en los últimos años en Santiago de Cali. En síntesis, a través de la creación de un programa curricular se busca motivar a la comunidad infantil en el reconocimiento y la valoración del espacio geográfico como constitutivo fundamental para la humanización y democratización de la sociedad.

\section{Objetivo}

Dada la gran importancia del proceso de formación docente en la primera infancia para el fomento del sentido de pertenencia y arraigo de la ciudad, en pro del desarrollo de competencias ciudadanas desde la edad temprana, el objetivo de la investigación es diseñar una estrategia praxeológica para la formación docente en la primera.

Figura 1. Árbol problematizador

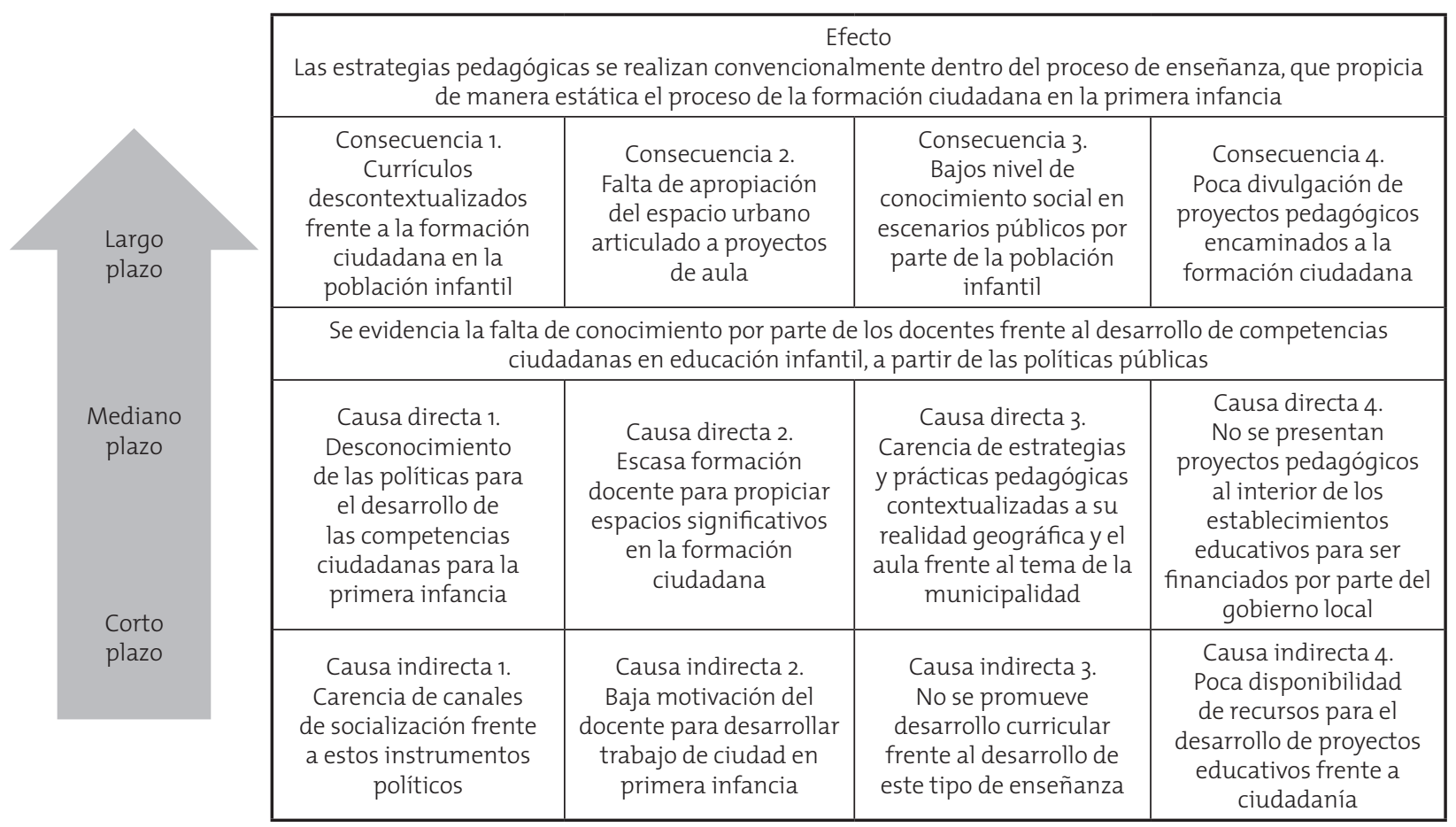

Fuente: elaboración propia. 


\section{Contexto histórico desde la educación inicial}

Aquí se pretende proporcionar el conocimiento general de la teoría que da significado a la investigación, debido a que presenta unos antecedentes teóricos que son estudios yo investigaciones realizadas sobre el tema. También, se muestra un mapeo conceptual que facilita el manejo teórico de la investigación con su respectiva argumentación textual.

\section{Antecedentes epistemológicos o estado del arte de la investigación}

Especialistas en el tema de enseñanza y aprendizaje desde la edad temprana llegan siempre a lo mismo: que el pedagogo debe propiciar espacios adecuados y estrategias pedagógicas innovadoras para desarrollar ciertas habilidades y destrezas en el infante, a través de la lúdica y la didáctica.

Pero a la hora de evaluar el desarrollo de este tipo de competencias en los niños y niñas, de manera notoria se evidencia una desarticulación de estos conocimientos adquiridos previamente para ser aplicados en la solución o resolución de problemas de la cotidianidad (Ministerio de Educación Nacional, 2006e, p. 8).

La evolución histórica de la educación inicial en Latinoamérica se puede traducir en el dinamismo que tiene no solo la familia, sino todos los sectores de la sociedad frente al desarrollo de las diversas etapas de crecimiento de los individuos. Lo que motivó la realización de la presente investigación es el compromiso social que tiene la educación, en especial la educación inicial en temas de formación ciudadana. Lo que se pretende mostrar a continuación es el estado del arte de las políticas públicas, programas y proyectos encaminados a la primera infancia, entendida esta como un proceso que ha contado con la participación de las entidades gubernamentales y de la sociedad civil, desde la década de los sesenta hasta la actualidad (tabla 1).

\section{Proceso de formación docente desde la praxeología}

Para facilitar el manejo del tema central de la investigación se plantea un cuadro sinóptico (figura 2). En diversas literaturas se habla sobre las dinámicas y el aporte que han tenido las ciencias sociales, entendidas como las ciencias aplicadas de la educación desde el enfoque social, a partir de las prácticas convergentes con otras ciencias, como la psicología y la sociología (Fermoso, 2003, p. 70). Diversos pensadores han contribuido en la evolución epistemológica de la praxeología, como Bourdieu, Boudon y Fermoso en Europa; y en Latinoamérica, Fals Borda, a través de procesos de investigación desde una perspectiva social a finales del siglo pasado.

Para el caso específico de la pedagogía social, que se encuentra inmersa dentro de las dinámicas de la educación social como ciencia, equivalente en ciertos aspectos al concepto de pedagogía praxeológica, estas pedagogías sin pretensiones exageradas, presentan un altísimo grado de correlación, en donde la primera es concebida como un paradigma o un nuevo modelo de educación en el quehacer pedagógico pensado como trabajo social (Fermoso, 2003, p. 72) y en la gestión educativa; mientras que la segunda se entiende como aquellas prácticas de transformación y adaptación al medio que tiene el individuo para el desarrollo de prácticas rutinarias, inducidas por agentes sociales (Bourdieu y Wacquart, 2005, p. 36). Entonces, se creería que el ideal de la propuesta praxeológica es una utopía para el cambio de los actuales modelos de educación, tal como hace referencia una obra homónima de Thomas More titulada Utopía (1516). Este título hace referencia a una isla y a la comunidad ficticia que la habita, cuya organización política, económica y cultural contrasta en numerosos aspectos con la sociedad inglesa de la época. Con esta obra More crea el género de las utopías políticas, y por ello, en términos más generales, esta palabra es empleada para referirse a una sociedad política ideal (figura 3). 
Tabla 1.

Evolución histórica de la educación inicial (datos recabado por el autor)

\begin{tabular}{|c|c|c|}
\hline \multirow[b]{2}{*}{ Década } & \multicolumn{2}{|r|}{ Hechos destacados } \\
\hline & $\begin{array}{c}\text { Contexto } \\
\text { internacional }\end{array}$ & Contexto nacional \\
\hline 60 & & $\begin{array}{l}\text { 1. Creación del Instituto Colombiano de Bienestar Familiar (ICBF) mediante la Ley } 75 \text { de } 1968 . \\
\text { 2. Creación de los Jardines Infantiles Nacionales por parte del MEN (1962). }\end{array}$ \\
\hline 70 & & $\begin{array}{l}\text { 3. Creación de los Centros de Atención Integral al Preescolar (CAIP), mediante la Ley } 27 \text { de } 1974 . \\
\text { 4. Inclusión de la educación preescolar como el primer nivel del sistema educativo formal } \\
\text { por parte del Ministerio de Educación Nacional, a través del Decreto o88 de } 1976 . \\
\text { 5. Diseño de la Política Nacional de Atención al Menor, que enfoca la atención del menor } \\
\text { de siete años atendiendo la situación de la salud y los procesos de socialización (Plan } \\
\text { de Integración Social, 1978-1982). } \\
\text { 6. Creación del Sistema Nacional de Bienestar Familiar (SNBF) mediante la Ley } 7 \text { de 1979, } \\
\text { que establece las normas para proteger a los niños y las niñas, la integración familiar, } \\
\text { garantizar los derechos del niño y de la niña y ejercer funciones de coordinación de } \\
\text { las entidades estatales. }\end{array}$ \\
\hline 80 & $\begin{array}{l}\text { Convención sobre los } \\
\text { Derechos del Niño } \\
\text { (Naciones Unidas, } \\
\text { 1989) }\end{array}$ & $\begin{array}{l}\text { 7. ElMEN implementa el Plan de Estudios para la Educación Preescolar con una concepción } \\
\text { de atención integral a la niñez y con la participación de la familia y la comunidad } \\
\text { (Decreto } 1002 \text { de 1984/ Plan de Desarrollo "Cambio con Equidad en 1982-1986"). } \\
\text { 8. Implementación del Programa de Hogares Comunitarios de Bienestar (HCB), } \\
\text { mediante los cuales se brindaría cuidado diurno, alimentación, atención básica en } \\
\text { salud y educación preescolar a los menores de siete años (ICBF, 1986). }\end{array}$ \\
\hline 90 & & $\begin{array}{l}\text { 9. La Constitución Política de 1991, en su artículo 67, establece que la educación será } \\
\text { obligatoria entre los cinco y los quince años de edad y comprenderá como mínimo } \\
\text { un año de preescolar. } \\
\text { 10. Creación del Programa Nacional de Acción en Favor de la Infancia a menores de } 18 \\
\text { años (PAFI), que retoma los planteamientos de la Cumbre de Jomtien (199o). } \\
\text { 11. Creación de los Jardines Comunitarios con los que se brinda atención a los niños y } \\
\text { niñas en poblaciones vulnerables, con la participación de los padres y acudientes } \\
\text { (ICBF mediante el Acuerdo } 19 \text { de 1993). } \\
\text { 12. Creación del Programa Grado Cero que busca ampliar la cobertura, elevar la calidad y } \\
\text { contribuir al desarrollo integral y armónico de todos los niños y niñas de cinco y seis } \\
\text { años de edad (con los sectores de salud, el ICBFy el MEN - Ley 115 de 1994). } \\
\text { 13. Formulación del CONPES } 2787 \text { de 1995, como una política pública sobre la infancia } \\
\text { "El tiempo de los niños", el cual es aprobado para contribuir al desarrollo integral de } \\
\text { los niños y de las niñas más pobres y vulnerables (programas de nutrición, salud y } \\
\text { educación). } \\
\text { 14. Ejecución de la estrategia del Pacto por la Infancia, como mecanismo para } \\
\text { descentralizar el PAFl y asegurar su ejecución a nivel local (Consejería para la Política } \\
\text { Social de la Presidencia de la República y DNP, 1996). } \\
\text { 15. Establecimiento de normas relativas a la organización del servicio educativo y } \\
\text { orientaciones curriculares del nivel preescolar (MEN, Decreto } 2247 \text { de 1997). En 1999 se } \\
\text { publican los lineamientos pedagógicos de este nivel. }\end{array}$ \\
\hline
\end{tabular}




\begin{tabular}{|c|c|c|}
\hline \multirow[b]{2}{*}{ Década } & \multicolumn{2}{|r|}{ Hechos destacados } \\
\hline & $\begin{array}{c}\text { Contexto } \\
\text { internacional }\end{array}$ & Contexto nacional \\
\hline $\begin{array}{c}2000- \\
2010\end{array}$ & $\begin{array}{c}\text { Ratificación por parte } \\
\text { de Colombia de la } \\
\text { Convención sobre los } \\
\text { Derechos de los Niños } \\
2000 \text { - Naciones } \\
\text { Unidas, Oficina del } \\
\text { Alto Comisionado } \\
\text { para los Derechos } \\
\text { Humanos } \\
\text { Cumbre del Milenio } \\
\text { en septiembre de } \\
\text { 20oo } \\
\text { Campaña del Milenio } \\
\text { de las Naciones } \\
\text { Unidas } \\
\text { Compromiso } \\
\text { hemisférico por } \\
\text { la educación de la } \\
\text { Primera Infancia - } \\
\text { 20o7 OEA y Consejo } \\
\text { Interamericano para } \\
\text { el Desarrollo Integral } \\
\text { (CIDI) } \\
\text { Reunión de alto nivel } \\
\text { sobre los Objetivos de } \\
\text { Desarrollo del Milenio } \\
\text { 20o8 }\end{array}$ & 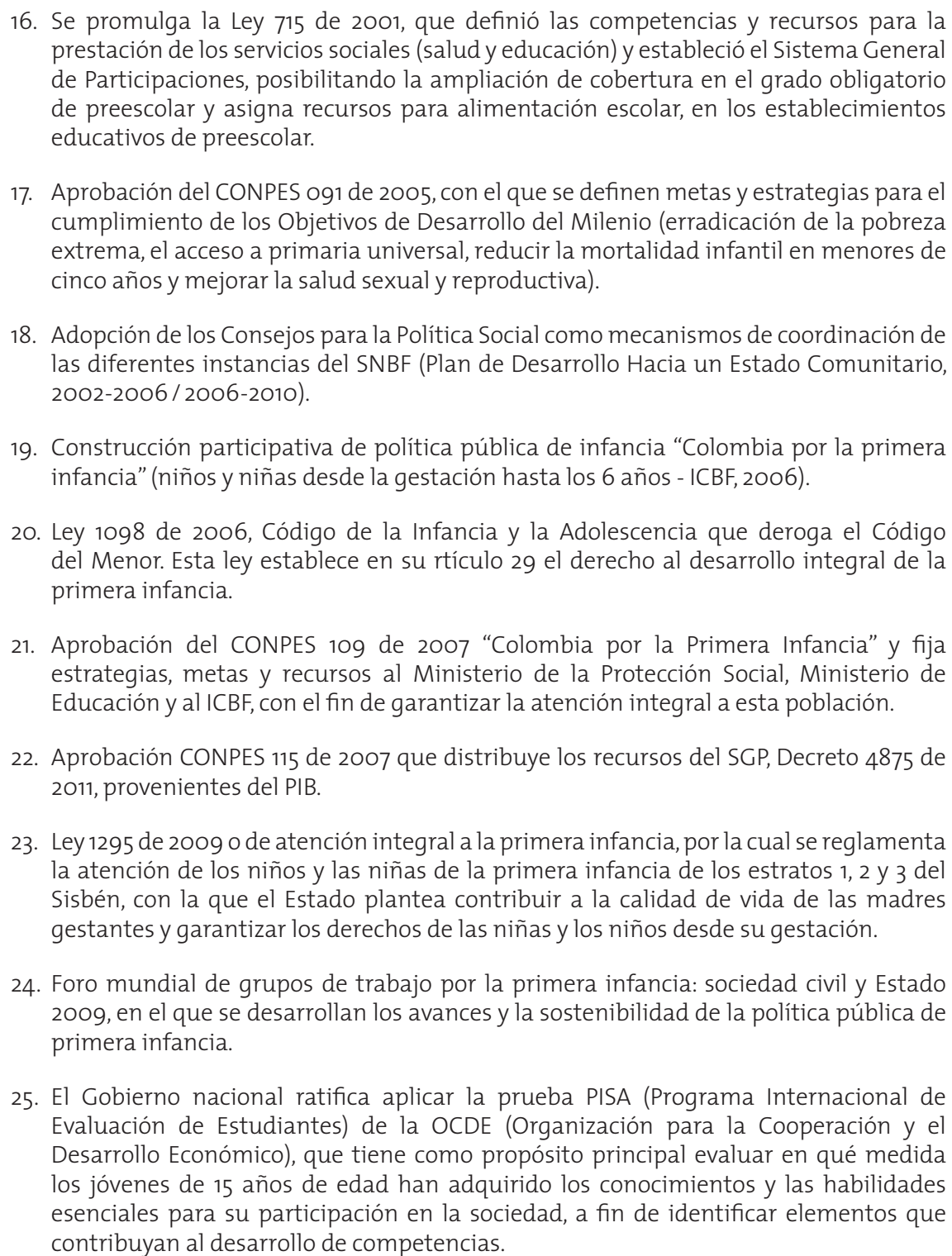 \\
\hline $\begin{array}{l}2010 \text { al } \\
\text { día de } \\
\text { hoy }\end{array}$ & $\begin{array}{l}\text { Cumbre Mundial } 2010 \\
\text { sobre los Objetivos de } \\
\text { Desarrollo del Milenio }\end{array}$ & $\begin{array}{l}\text { 26. Plan de Desarrollo: Prosperidad para todos (2010-2014) } \\
\text { 27. Distribución de los Recursos del Sistema General de Participaciones (Conpes Social 152) } \\
\text { 28. Recursos para la Atención Integral de la Primera Infancia Conpes } 162 \text { (el MEN crea el } \\
\text { Fondo de Fomento a la Atención en convenio con ICETEX). } \\
\text { 29. Decreto por el cual se crea la Comisión Intersectorial para la Atención Integral de la } \\
\text { Primera Infancia, Decreto } 4875 \text { de } 2011 . \\
\text { 30. Se publica el documento Fundamentos Políticos, Técnicos y de Gestión de la estrategia } \\
\text { de atención integral a la primera infancia, } 2013 \text {. }\end{array}$ \\
\hline
\end{tabular}


Figura 2. Cuadro sinóptico sobre el proceso de formación docente desde la praxeología

Educación social desde el enfoque praxeológico

\begin{tabular}{|c|c|c|c|}
\hline \multirow[b]{2}{*}{ Definiciones } & \multicolumn{2}{|c|}{$\begin{array}{l}\text { Proceso de formación } \\
\text { docente a distancia }\end{array}$} & \multirow[b]{2}{*}{$\begin{array}{l}\text { Desarrollo de } \\
\text { competencias }\end{array}$} \\
\hline & Características & $\begin{array}{c}\text { Agentes de } \\
\text { acompañamiento }\end{array}$ & \\
\hline $\begin{array}{l}\text { Métodos de } \\
\text { enseñanza }\end{array}$ & $\begin{array}{l}\text { Proceso de } \\
\text { enseñanza }\end{array}$ & Profesores & $\begin{array}{l}\text { En el } \\
\text { profesor }\end{array}$ \\
\hline $\begin{array}{l}\text { Métodos de } \\
\text { autoformación }\end{array}$ & $\begin{array}{l}\text { Proceso de } \\
\text { autoformación }\end{array}$ & Estudiantes & 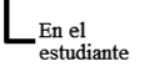 \\
\hline $\begin{array}{l}\text { Ledagogía de } \\
\text { reflexión social }\end{array}$ & & $\begin{array}{l}\text { Directivas y } \\
\text { académicos }\end{array}$ & \\
\hline
\end{tabular}

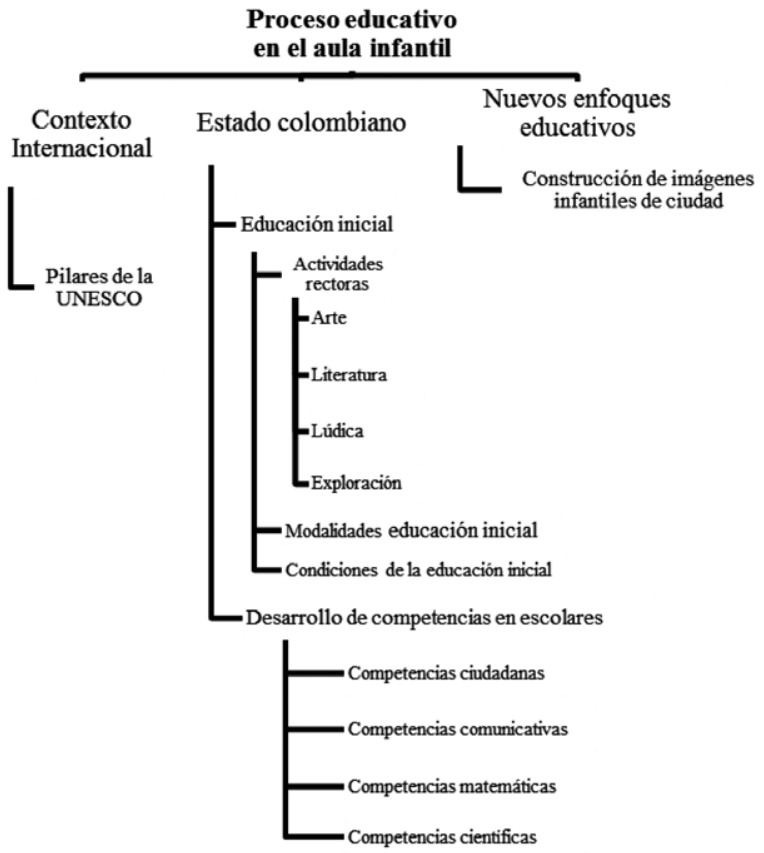

Fuente: elaboración propia.

Figura 3. Representación utópica de Robert Owen (1838)

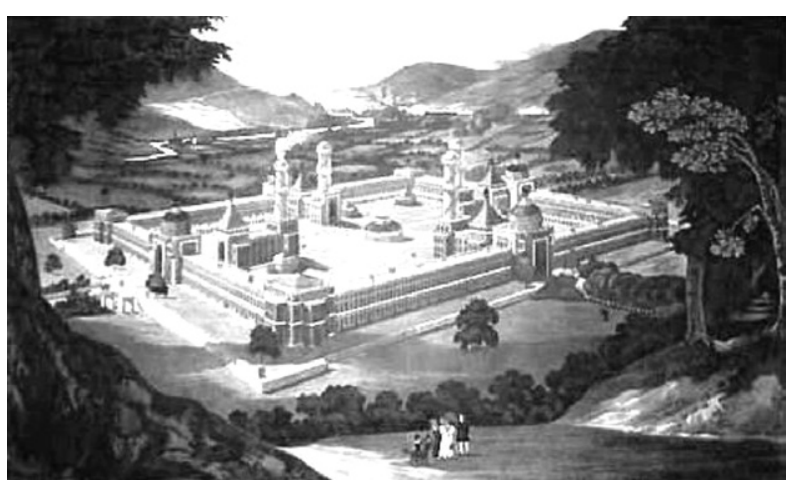

Un ejemplo de educación social es la Corporación Universitaria Minuto de Dios - Uniminuto, institución que contribuye al desarrollo humano sostenible, promoviendo en la comunidad académica la producción de trabajo investigativo alrededor de un modelo educativo de interacción social, experi- mentado con más de sesenta años en trabajo social. Desde la misión institucional ${ }^{2}$ tiene concebido favorecer a comunidades diversas ofreciendo educación de calidad, garantizando el acceso y la permanencia dentro de un sistema educativo acompañado de una perspectiva humanística para la transformación social y el desarrollo sostenible. Para garantizar la calidad de los procesos, Uniminuto está desarrollando a cabalidad todo lo que contempla la autoridad nacional para educación superior, a través de un sistema

2 Sistema Universitario Uniminuto inspirado en el Evangelio, el pensamiento social de la Iglesia, la espiritualidad Eudista y el carisma del Minuto de Dios, tiene como propósito: ofrecer educación superior de alta calidad y pertinente con opción preferencial para quienes no tienen oportunidades de acceder a ella, a través de un modelo innovador, integral y flexible. Formar excelentes seres humanos, profesionales competentes, éticamente orientados y comprometidos con la transformación social y el desarrollo sostenible. Contribuir, con nuestro compromiso y nuestro testimonio, a la construcción de una sociedad fraterna, justa, reconciliada y en paz. 
de gestión de calidad institucional. Esto garantiza la aplicación del modelo praxeológico que, en últimas, no pretende desarrollar metodologías innovadoras a partir de teoría, sino identificar los contenidos a partir de la lectura de los contextos socioculturales.

\section{El concepto de la educación a distancia como modalidad de educación social}

Se evidencia la necesidad de encontrar una serie de denominaciones, estructuras, metodologías, organización, uso de tecnologías, tamaño de la institución, demandas diferenciadas y proyectos educativos institucionales, además de todos los sistemas frente a lo fáctico de la enseñanza a distancia, los cuales suelen tener en común una serie de características que son las fuentes y los autores que han trabajado esta cuestión a lo largo de la historia.

Con el fin de clarificar el concepto de educación a distancia, es aconsejable ofrecer las siguientes definiciones o los rasgos característicos que han sido asignados por estudiosos dentro de esta.

Para iniciar, se parte del término educación a distancia, que cubre un amplio espectro de diversas formas de estudio y estrategias educativas, que tienen en común que no siguen la tradicional modalidad presencial; una de estas estrategias es la interacción entre profesores y alumnos en sus espacios de diálogo (Casas, 1982, en García, 1987, p. 1).

En el estudio a distancia el aprendizaje está canalizado con ayuda de medios técnicos (Fritsch, 1984, en García, 2001, p. 12). Por otro lado, la enseñanza y educación a distancia es un método de impartir conocimientos, habilidades y actitudes, racionalizando mediante la aplicación de la división del trabajo y de principios organizativos, así como por el uso extensivo de medios técnicos, especialmente para el objetivo de reproducir material de enseñanza de alta calidad, lo cual hace posible instruir aún gran número de estudiantes al mismo tiempo y donde quiera que ellos vivan. Es una for- ma industrial de enseñar y aprender (Peters, 1983, en García, 2001, p. 13).

Sarramona (1991, p. 199) la señala como una metodología de enseñanza donde las tareas docentes acontecen en un contexto distinto de las discentes, de modo que estas resultan, respecto de las primeras, diferidas en el tiempo, en el espacio o en ambas dimensiones a la vez.

\section{La pedagogía praxeológica como práctica social en la educación a distancia}

La praxeología se encuentra frente a tres niveles lógicos: la lógica de los actores (decisiones, hábitos, racionalidades), la de las situaciones (interacciones, juegos, dramas) y la lógica de la explicación misma (Juliao, 2002, p. 45). Esta última trata de la praxis, entendida como la unidad dinámica y dialéctica entre la práctica social y su pertinente análisis y comprensión teórica; es decir, trata de la relación entre la experiencia y la transformación y la teoría que ayuda a conducir la acción.

La explicación praxeológica de la actividad social, por tanto, parte de analizar las lógicas de los actores concretos y pretende reconstruir objetivamente su realidad social. En el terreno de la pedagogía y la educación también parecen existir dos concepciones opuestas de la praxeología y su uso. En la primera, sirve de aval ideológico a las prácticas normativas que buscan integrar a las personas en el sistema productivo. Se garantiza la gestión óptima de los recursos humanos y es, al mismo tiempo, un modo de legitimación y un procedimiento manipulador de real eficacia. La segunda, en oposición a la precedente, asigna como propósitos de la praxeología el discernimiento de las prácticas y los proyectos individuales o colectivos, para facilitar su realización en un contexto determinado.

Con lo expuesto, se puede reconocer la intrínseca articulación entre la experiencia de investigación en el aula y en su mismo proceso formativo entre el agente (profesor) y el sujeto de estudio (estudiante). 
Se manifiesta así la puesta en marcha de un trabajo mancomunado comunitario, participativo e incluyente entre aquellos actores sociales para desarrollar procesos de educación social que ayuden a la resolución de problemas de una sociedad. Por ende, el modelo educativo de Uniminuto está ligado a la labor comunitaria, a través de la pedagogía social como metodología de investigación social.

\section{Proceso educativo social en el aula infantil incluyente}

Se entiende que todo individuo se encuentra en constante proceso de educación desde sus primeros años de vida, y es ahí donde se entra a definir el concepto de educación inicial. Es un derecho impostergable de la primera infancia, la cual se constituye en un estructurante de la atención integral cuyo objetivo es potenciar de manera intencionada el desarrollo de las niñas y los niños desde su nacimiento hasta cumplir los seis años, partiendo del reconocimiento de sus características y de las particularidades de los contextos en que viven, y favoreciendo interacciones que se generan en ambientes enriquecidos a través de experiencias pedagógicas y prácticas de cuidado (Ministerio de Educación Nacional, 2014a, p. 5). La educación inicial es válida en sí misma por cuanto el trabajo pedagógico que allí se planea parte de los intereses, inquietudes, capacidades y saberes de las niñas y los niños. Esta no busca como fin último su preparación para la escuela primaria, sino que les ofrece experiencias retadoras que impulsan su desarrollo; allí juegan, exploran su medio, se expresan a través del arte y disfrutan de la literatura (MEN, 2014a, p. 10).

Proceso de enseñanza y aprendizaje en la educación inicial. Anijovich y Mora (2009) señalan que, en la educación inicial, los agentes educativos deben crear e innovar estrategias de enseñanza que sean múltiples y variadas para que los niños y las niñas se desarrollen integralmente en condiciones perfectas, fomentando la curiosidad para que aprendan y apliquen sus conocimientos en situaciones cotidianas. Todo esto debe fortalecer la capa- cidad de comunicarse eficazmente con otros pares y miembros de la sociedad, y por ende con la participación de la familia para que desarrollen adecuados hábitos de trabajo y se preparen para hacer la transición al mundo escolar.

Proceso de enseñanza infantil. El proceso de enseñanza produce un conjunto de transformaciones sistemáticas en los individuos. Implica una serie de cambios graduales cuyas etapas suceden en orden ascendente. Los padres de familia y docentes deben comunicarse frecuentemente para discutir los intereses del niño y la niña, así como su desarrollo. Cada niño es único y, por tanto, la mejor forma de comprender su entorno depende de su aptitud innata y el nivel de interés que demuestran en determinadas áreas del conocimiento. Lo que los agentes educativos deben hacer es alimentar, desarrollar y perfeccionar una aptitud e interés creando una atmósfera que aliente el aprendizaje, mediante la implementación de actividades que sean efectivas e innovadoras en los niños y las niñas.

Fases del proceso de enseñanza. En el proceso de enseñanza se pueden distinguir fases o etapas, cada una de ellas se diferencia en cuanto a sus propósitos, y, por ende, en las actividades que las conforman y realiza el docente (Torres, 1994, p. 23):

- $\quad$ Las que se vinculan con el planeamiento: el agente educativo propone objetivos, selecciona y organiza los contenidos pertinentes, prevé actividades formativas y materiales curriculares teniendo en cuenta intereses y habilidades propias para que el aprendiz se apropie de ese saber y desarrolle las capacidades propuestas en los objetivos.

- Las que se relacionan con la gestión del proceso de enseñanza: el docente explora saberes y experiencias previamente adquiridos por los aprendices, y que se relacionan con los nuevos contenidos; orienta el diálogo y reflexión grupal, organiza y supervisa la práctica, propone trabajos integradores (problemas, 
casos, proyectos); orienta la reflexión sobre la práctica realizada y la síntesis de conocimientos y métodos aprendidos.

- Las que se vinculan con la evaluación del proceso y de los resultados. La evaluación puede ser definida como el proceso de obtener información que sirve de base para juzgar el valor de las actividades realizadas y los resultados de aprendizaje alcanzados.

Proceso de aprendizaje infantil. El proceso de enseñanza genera un aprendizaje, entendiéndose como un proceso natural, extremadamente complejo, cuya esencia es la adquisición de un nuevo conocimiento, habilidad o capacidad en el individuo. Para que dicho proceso pueda considerarse realmente como aprendizaje, en lugar de una simple huella o retención pasajera, debe poder manifestarse en un tiempo futuro. Además, debe contribuir a la solución de problemas concretos, incluso diferentes en su esencia a los que motivaron inicialmente el desarrollo del conocimiento, habilidad o capacidad.

El aprendizajetambién se puede definir como un proceso de cambio relativamente permanente en el comportamiento de una persona, generado por la experiencia (Feldman, 2008, p. 271). En primer lugar, aprendizaje supone un cambio conductual, lo cual implica que se desarrolle una interacción entre el sujeto y el ambiente a través de la experiencia. En segundo lugar, el aprendizaje produce un cambio cognitivo, se basa en el conocimiento previo, involucrando la asimilación y acomodación de experiencias; así se obtiene una nueva información o un nuevo conocimiento. Existen diferentes tipos de aprendizaje, para Torres (1994, p. 23) estos son:

- Aprendizaje receptivo, en este tipo de aprendizaje el sujeto solo necesita comprender el contenido para poder reproducirlo, pero no descubre nada.

- $\quad$ Aprendizaje repetitivo o memorístico, se produce cuando el alumno memoriza contenidos sin comprenderlos o relacionarlos con sus conocimientos previos, y por ende no encuentra significado a los contenidos.

- Aprendizaje por descubrimiento, aquí el sujeto no recibe los contenidos de forma pasiva; solamente descubre los conceptos y sus relaciones, además los reordena para adaptarlos a su esquema cognitivo.

- $\quad$ Aprendizaje significativo es aquel en el cual el sujeto relaciona sus conocimientos previos con los nuevos, dotándolos así de coherencia respecto a sus estructuras cognitivas.

Pilares básicos de la educación promulgados por la Organización de las Naciones Unidas para la Educación, la Ciencia y la Cultura (Unesco). La educación es un instrumento indispensable para que la humanidad pueda progresar hacia los ideales de paz, libertad y justicia social (Delors, 1997, p. 9). Constituyéndose esta no solo en parte fundamental para el desarrollo económico y político de la sociedad, sino también el vehículo que permite potenciar y desarrollar estrategias en la educación inicial, base fundamental para el desarrollo del individuo, planteado a través de cuatro pilares básicos que pretende formar un ser integral, que permita aprender a conocer, hacer, vivir juntos y ser.

Pilar 1. Aprender a conocer: implica necesariamente aprender a aprender, aprender a comprender el medio que lo rodea, ejercitando la memoria y el pensamiento, como instrumentos para comprender. Así, ejercitar la concentración de la atención en las cosas y en las personas implica un proceso de descubrimiento que requiere la permanencia y profundización de la información captada que puede provenir de situaciones y eventos cotidianos o provocados.

Pilar 2. Aprender a hacer: aprender a conocer y aprender a hacer son, en gran medida, indisociables. Lo que requiere de unas cualidades humanas subjetivas innatas o adquiridas que correspondan al establecimiento de relaciones estables y eficaces 
entre las personas que les permitan influir sobre su propio entorno, hacer frente a diversas situaciones, trabajar en equipo, cooperar, ser tolerante, respetuoso y, además, la potenciación de aprendizajes verdaderamente significativos en situaciones que tienen valor especial para el que aprende a través de la acción, el intercambio de información con los demás, la toma de decisiones y puesta en práctica de lo aprendido.

Pilar 3. Aprender a vivir juntos: requiere partir del reconocimiento de sí mismo - ¿quién soy?-como persona o como institución, para poder realmente ponerse en el lugar de los demás y comprender sus reacciones. En este sentido, la educación tiene una doble misión: de un lado, el descubrimiento del otro, que enseña sobre la diversidad de la especie humana y contribuye a una toma de conciencia de las semejanzas, las diferencias y la interdependencia entre todos los seres humanos.

Pilar 4. Aprender a ser: requiere que todos los seres humanos estén en condiciones de dotarse de un pensamiento autónomo, crítico y elaborar un juicio propio para determinar por sí mismos qué deben hacer en las diferentes circunstancias de la vida. Por ello, la educación debe propiciar la libertad de pensamiento, juicio, sentimientos e imaginación para que sus talentos alcancen la plenitud y, en lo posible, sean artífices de su destino.

En el contexto colombiano, el Ministerio de Educación Nacional (2014a, p. 30) establece el desarrollo en la población infantil del conocimiento, los valores y las competencias necesarias para el bienestar personal y familiar a partir de:

- Uno mismo es el responsable principal de la definición de resultados positivos para el futuro.

- Estimular el descubrimiento y la experimentación.

- Adquirir valores compartidos universalmente.

- Desarrollar una personalidad y una identidad propia, el conocimiento de sí mismo y la autorrealización.
- $\quad$ Ser capaz de actuar con mayor autonomía, fundamento y responsabilidad personal.

De igual manera, el MEN (2014a, p. 23) señala que el camino para crear una cultura de educación inicial pensada desde lo social, a través de todos los esfuerzos de la comunidad educativa y la sociedad civil, pensando en el marco de la atención integral, son las cuatro actividades rectoras promulgadas por la autoridad de educación a nivel nacional: el juego, el arte, la literatura y la exploración del medio. Los pilares de la educación son un grupo de soportes que integran las relaciones iniciales que el niño tiene con el mundo, en su contexto, sea este familiar o institucional.

Dentro de los lineamientos pedagógicos de educación inicial nacional del MEN (2014a, p. 35) se plantean como elementos fundamentales en el trabajo pedagógico con los niños el juego, el arte, la literatura y la exploración del medio como actividades rectoras de la primera infancia; lejos de ser herramientas o estrategias pedagógicas, que se usan como medio para lograr otros aprendizaje, en sí mismas posibilitan aprendizajes.

El juego: es un reflejo de la cultura y la sociedad, y en él se representan las construcciones y los desarrollos de los entornos y sus contextos. Las niñas y los niños juegan a lo que ven y al jugar lo que viven resignifican su realidad. Por esta razón, el juego es considerado como medio de elaboración del mundo adulto y de formación cultural, que inicia a los pequeños en la vida de la sociedad en la cual están inmersos. En el juego hay un gran placer por representar la realidad vivida de acuerdo con las propias interpretaciones, y por tener el control para modificar o resignificar esa realidad según los deseos de quien juega (MEN, 2014C, p. 64).

La literatura: la necesidad de comunicación es innata en el desarrollo del ser, se utilizan diferentes formas para comunicar necesidades y gustos, desde el primer llanto al nacer se pretende tener una relación con la otra persona para poder expresar lo que se siente. El acercamiento al lenguaje verbal 
permite en los niños y las niñas no solo una relación con lo escrito sino una construcción de palabras que llevan al sujeto al juego, a darle un sentido según sea la experiencia vital de cada individuo (MEN, 2014d).

$\mathrm{El}$ arte: la expresión artística en la primera infancia permite el buen desarrollo de las múltiples capacidades y habilidades que el niño presenta desde que nace, como la creatividad, la expresión y la autonomía. Su desarrollo se construye a través de las relaciones con el medio, con su entorno y con el mundo de los objetos, lo cual abre paso a la construcción de un pensamiento lógico. El arte permite que ese pensamiento, aún no real en sus primeros años, sea expresado y con gran intención de ser comunicado para dar a conocer una idea, la idea de cómo el niño ve el mundo a través de su experiencia y de cómo la comunicación puede ser significativa para establecer las cosas del medio con las que más se identifica (MEN, 2014b, p. 21).

La exploración del medio: el pilar de la exploración del medio se piensa desde el aprendizaje de la vida y todo lo que está a su alrededor; es aprender a conocer los objetos físicos naturales y los construidos por el hombre, las personas, las relaciones entre unos y otras, los fenómenos naturales; es empezar a entender que lo social y lo natural están en permanente interacción (MEN, 2014e, p. 104).

Las niñas y los niños llegan a un mundo construido, donde todo es físico, biológico, social y cultural, al que necesitan adaptarse y que los necesita para transformarse. En él encuentran elementos y posibilidades para interactuar gracias a sus propias particularidades y capacidades. Los sentidos — gustar, tocar, ver, oler, oír- cumplen un papel fundamental en la exploración por cuanto sirven para aproximarse al medio de diversas maneras. Explorar permite a las niñas y los niños acercarse a las construcciones sociales, al tiempo que se apropien de su cultura desde la convivencia en lo cotidiano (MEN, 2006e, p. 17). De igual manera, permite cuestionarse, resolver problemas, interactuar, usar su cuerpo, investigar, conocer, ensayar, perseverar, ganar independencia. Esta experiencia implica un proceso de construcción de sentido acerca de lo que pasa en el mundo y de lo que significa ser parte de él.

El juego, la literatura, el arte y la exploración del medio son actividades en muchos casos interdependientes. Así, por ejemplo, cuando la niña o el niño juega o explora, puede hacerlo manipulando materiales plásticos como pinturas o arcillas, con lo cual se acerca a una experiencia artística. También hay casos en donde el juego se combina con la expresión literaria y musical.

\section{Metodología}

En este apartado se reconoce el tipo y método de la investigación. También, la ubicación donde se va a realizar; todo lo relacionado al registro y sistematización de los datos, tanto primarios, como secundarios; la población y la muestra que ayuda a la comprensión del objeto de estudio y la unidad de análisis.

\section{Tipo y método de investigación}

El método que se emplea en esta propuesta es la investigación acción participación (IAP), cuyo método de estudio apunta a la producción de un conocimiento propositivo y transformador, mediante un proceso de debate, reflexión y construcción colectiva de saberes entre los diferentes actores de un entorno, con el fin de lograr transformación social en el proceso de formación docente en el contexto de la educación infantil.

Frente al tipo de investigación se radica de manera mixta, es decir, cualitativa y al mismo tiempo cuantitativa, lo cual significa un determinado enfoque de producción de conocimientos sistemáticos, orientado a la comprensión a profundidad de los fenómenos, a la transformación de prácticas en escenarios educativos no convencionales, y proponer la toma de decisiones, implicando una preocupación directa por las situaciones de interés tal como son vividas, sentidas y experimentadas. Lo anterior 
significa que se busca obtener resultados fiables y útiles para mejorar situaciones colectivas, con base en un análisis crítico, con la participación activa de los grupos implicados, que se orienta a estimular la práctica transformadora y el cambio social. La propuesta educativa está encaminada a fomentar y desarrollar en la comunidad estudiantil y académica competencias de carácter comunicativo, cognitivo, valorativo y práxicas. ${ }^{3}$

\section{Ubicación geográfica}

Esta investigación se lleva a cabo dentro del contexto de educación inicial municipal de la ciudad de Santiago de Cali, por lo cual la Corporación Universitaria Minuto de Dios, como institución de educación superior de carácter social, dirigida a estratos socioeconómicos menos favorecidos, promueve la presente investigación a través del programa de licenciatura en pedagogía infantil de la ciudad de Cali.

\section{Población y muestra}

En las investigaciones, tanto de enfoque cuantitativo, como cualitativo se debe determinar la unidad de análisis, la cual es definida por Hernández, Fernández y Baptista (2010) como los sujetos u objetos de estudio que van a ser medidos. Para este tipo de enfoque investigativo, se ratifica que el levantamiento de la información es de carácter primario, a través de unos instrumentos (más adelante se explica cuáles son los instrumentos que fueron empleados) como método.

En el presente estudio, coherente con el propósito enunciado en líneas anteriores, para la unidad de análisis se plantearon tres grupos: el grupo A, el cual está conformado por 600 docentes en formación de la licenciatura en pedagogía infantil de Uniminuto Cali. El grupo B, conformado por los 14 pro-

3 Entendidas estas como la conjugación e interiorización de la teoría, a través de espacios de aplicación, demostración y resolución de problemas sociales del entorno. fesores del programa académico. Para terminar, el grupo C está conformado por tres directivas académicas y administrativas de Uniminuto Valle (rector, vicerrector académico y directora administrativa).

Clarificada la unidad de análisis, se pasa a determinar la población de la investigación, entendida como el total de individuos que cumplen con las especificaciones antes indicadas. Para facilitar la recolección de datos y en aquellos casos en que la población es muy numerosa, los especialistas en estadística sugieren la escogencia de una muestra representativa, la cual es un subgrupo elegido entre todos los sujetos que hacen parte de la población. Para el presente estudio se requiere de muestreo, y a su vez se describe en los siguientes tres apartados (tabla 2).

Tabla 2. Determinación de la población y muestreo

\begin{tabular}{|c|c|c|c|}
\hline Grupo & Categorización & Población & Muestra \\
\hline A & $\begin{array}{c}\text { Docentes en } \\
\text { formación Uniminuto } \\
\text { Cali }\end{array}$ & 600 & 120 \\
\hline B & $\begin{array}{r}\text { Académicos Unidad de } \\
\text { Educación Uniminuto } \\
\text { Cali }\end{array}$ & 14 & 7 \\
\hline C & Directivas académicas & 05 & $\begin{array}{c}\text { No } \\
\text { aplica }\end{array}$ \\
\hline
\end{tabular}

Fuente: datos recabados por el autor.

\section{Fases o etapas del proyecto}

Este proyecto se desarrolla en cinco etapas, entendidas como el proceso de esta investigación, comprendidas de la siguiente manera:

Etapa 1: fundamentación teórica y contextualización del objeto de estudio. Dentro de esta etapa se efectúa todo lo concerniente a la conceptualización y contextualización del problema de investigación.

Etapa 2: caracterización de la población objeto de estudio. A través de instrumentos de registro de datos e información, como los resultados de las 
ISSN $0123-1294$ | e-ISSN 2027-5358 | Educ.Educ. Vol. 19. No.3 | Septiembre-Diciembre de 2016 | pp. 416-436.

Universidad de La Sabana | Facultad de Educación

evaluaciones cognitivas propias del proceso de titulación de educadores, encuestas y entrevistas dirigidas al cuerpo de profesores y directivas de Uniminuto. Todos los instrumentos para la recolección de datos contaron con un proceso de validación del conocimiento y constructo a través de parámetros estadísticos.

Etapa 3: diseño de la cartilla. Esta etapa se enfoca en diseñar el material didáctico dirigido a educadores escolares para el desarrollo de competencias ciudadanas en el aula.

Etapa 4: socialización del producto y material pedagógico. Para culminar con la investigación, se hace entrega de tres productos, el diseño de un ejemplar ilustrado a color como guía docente, y otro ejemplar para la población infantil. Y el último, será la sistematización de la experiencia, a través de una cartilla institucional.

\section{Resultados}

\section{Al interior de la academia}

Con el propósito de identificar el perfil de los profesores del Programa de Licenciatura en Pedagogía Infantil, se describen algunos de los espacios pedagógicos que se tienen disponibles dentro de la educación superior a distancia que faciliten el proceso pedagógico desde la praxeología.

\section{Insumos físicos}

La infraestructura física en Uniminuto rectoría Valle cuenta con:

- Dos auditorios con capacidad de 120 asistentes cada uno (figura 4).

- Una biblioteca con espacio para estudio y lectura.

- Más de sesenta aulas de clase para el desarrollo de encuentros tutoriales dentro del proceso de educación a distancia tradicional, las cuales se encuentran distribuidas en cuatro edificios (figura 5).
- Cuatro salones pedagógicos especiales, el salón de los espejos, el salón de multimedia y salón VIP (figuras 6 y 7 ).

- Un coliseo deportivo cubierto.

Figura 4. Espacio para la realización de plenarias

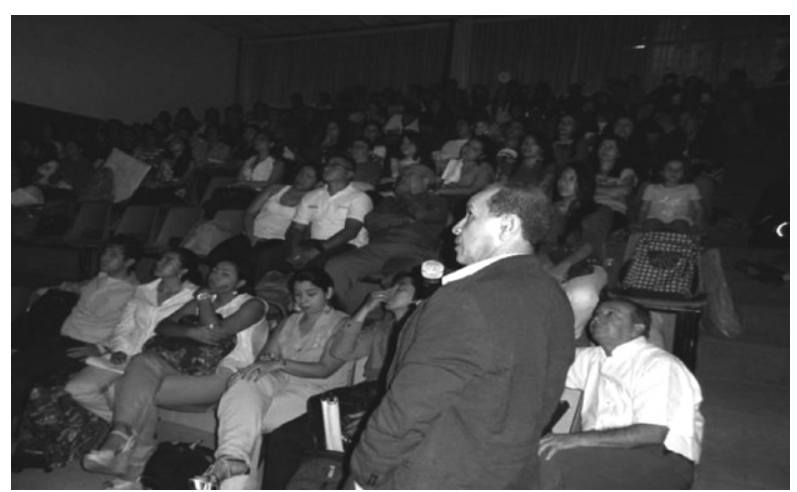

Fuente: foto del autor.

\section{Figura 5. Espacio para la realización de tutorías}

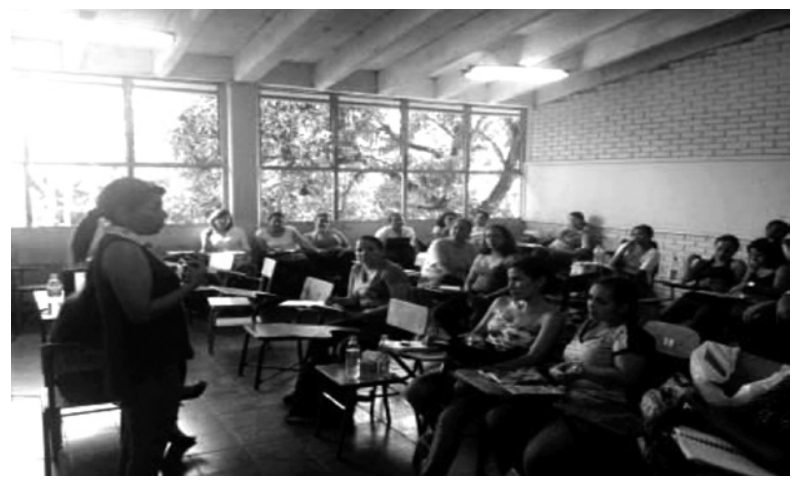

Fuente: foto del autor.

Figura 6. Salón Multimedia

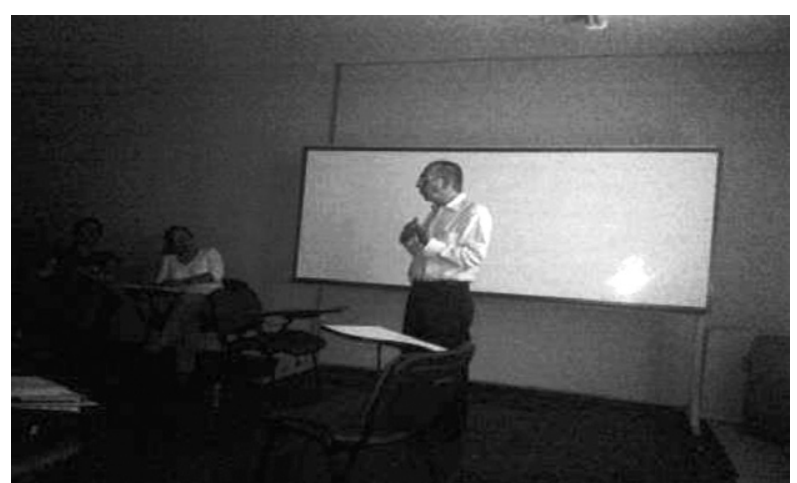

Fuente: foto del autor. 
Figura 7. Salón VIP

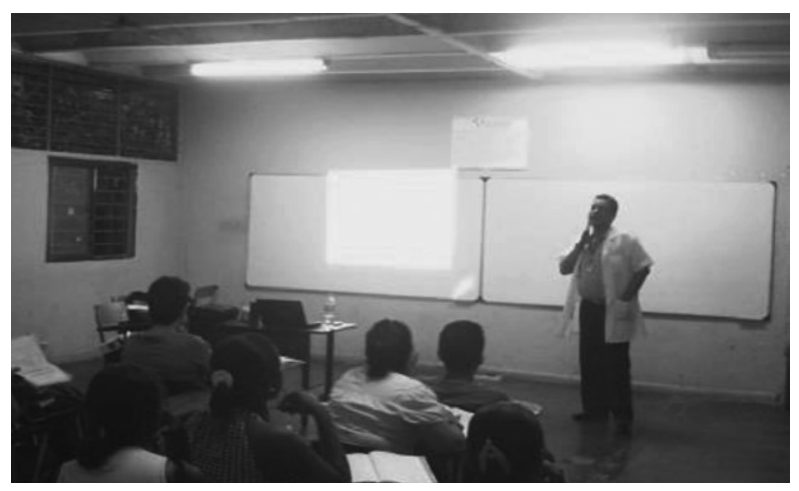

Fuente: foto del autor.

\section{Insumos de información y tecnología para la educación virtual}

La infraestructura tecnológica en Uniminuto rectoría Valle cuenta con:

- Una plataforma educativa moodle (para proceso de autoformación, acceso y consulta de bases de datos en línea).

- E-books.

Por otro lado, se realizó un estudio con ocho tutores hora cátedra del programa académico licenciatura en pedagogía infantil, a través de la realización de un cuestionario conformado por 16 preguntas semiabiertas y abiertas, las cuales tenían la intención de identificar sus percepciones frente al modelo praxeológico, características propias para desarrollar una sesión tutorial, la identificación del modelo pedagógico institucional, sus retos y habilidades para afrontar la globalización de las TIC en el contexto académico, entre otras.

\section{Para cerrar...}

En estas últimas líneas del artículo se concluye que la comunidad académica está conformada por estudiantes y el cuerpo de profesores que se ubican en el escenario del proceso de aprendizaje y enseñanza respectivamente en Uniminuto.

\section{De la comunidad estudiantil}

Este grupo se identifica por sus actitudes, comportamientos y sus hábitos de estudio al ingresar a este tipo de modalidad educativa, en donde se percibe que existe un aumento en el interés para participar en diversos eventos programados por parte de la institución, como del programa académico. Lo anterior se demuestra en el número de inscripciones de estudiantes al proceso de semilleros de investigación. Ahora, en el presente año fue un éxito la convocatoria que realizó la Licenciatura en Pedagogía Infantil para inscribir proyectos de diversa índole que fomenten la formación ciudadana desde la edad temprana, como mecanismo integrador de la educación social que caracteriza a Uniminuto, a través de la pedagogía praxeológica social.

\section{Los profesores, concebidos como colaboradores pedagógicos sociales}

En este grupo se muestran las condiciones de los profesores que son los encargados de poner en marcha el proceso de enseñanza, para alcanzar niveles de aprendizaje acordes en los estudiantes dentro de un contexto de práctica social, como lo fomenta el enfoque praxeológico.

En la medida que se incrementa la cultura de la pedagogía social en el cuerpo de profesores, a través de la articulación entre la fundamentación teórica con la práctica estudiantil, reflejados en el desarrollo de microcurrículos innovadores y en el aumento en la calidad educativa, los educadores tendrán la posibilidad de trascender en su quehacer en el trabajo social.

Otra de las condiciones que favorece este trabajo de carácter praxeológico en el quehacer pedagógico es la valoración de su labores sociales al interior de la institución, que además promueve el desarrollo de un modelo educativo abierto a toda la sociedad. 
ISSN $0123-1294$ | e-ISSN 2027-5358 | Educ.Educ. Vol. 19. No.3 | Septiembre-Diciembre de 2016 | pp. 416-436.

Universidad de La Sabana | Facultad de Educación

\section{Referencias}

Alcaldía de Santiago de Cali (2004). Plan decenal municipal de educación 2004-2014. Cali: DAPM.

Alcaldía de Santiago de Cali (2012). Plan de Desarrollo Municipal 2012-2015. Cali: DAPM.

Anijovich, R.y Mora, S. (2009). Estrategias de enseñanza: otra mirada al quehacer en el aula. Buenos Aires: Aique.

Bauman, Z. (2002). La cultura como praxis. Barcelona: Paidós.

Bourdieu, P. (2003). Capital cultural, escuela y espacio social. Buenos Aires: Prometeo.

Bourdieu, P.y Wacquart, L. (2005). Una invitación a la sociología reflexiva. Buenos Aires: Siglo XXI.

Carvajal, M. (2004). Percepción ambiental y construcción de lugar [monografía de grado]. Santiago de Cali, Colombia: Universidad Autónoma de Occidente.

Casas, L. (1994). Bases teóricas de la educación a distancia. Informe de Investigaciones Educativas, 8 (2), 11-43.

Congreso de la República de Colombia (1994). Ley general de educación-Educación Básica y Media Vocacional. Bogotá D.C.: Diario Oficial.

Corporación Universitaria Minuto de Dios (2013). Proyecto Educativo Institucional. Bogotá: Uniminuto.

De Castro, C. (1997). La geografía en la vida cotidiana: de los mapas cognitivos al prejuicio regional. Barcelona: Ediciones del Serbal.

Delors, J. (2006). La educación encierra un tesoro. Bogotá: Unesco.

Feldman, R. (2005). Autoestima ¿cómo desarrollarla? ( $3^{\mathrm{a}}$ ed.). Madrid: Narcea.

Fermoso P. (2003). ¿Pedagogía social o ciencia de la educación social? Pedagogía social, 10 (45), 61-84.

Freire, P. (2006). Pedagogía de la autonomía: saberes necesarios para la práctica educativa (11 ed.). Madrid: Siglo XXI.

García, L. (1987). Hacia una definición de educación a distancia. Boletín Informativo de la Asociación Iberoamericana de Educación Superior a Distancia, 4 (18), 1-9.

García, L. (2001). La educación a distancia: de la teoría a la práctica. Barcelona: Ariel.

Hernández, S. R., Fernández, C. y Baptista, P. (2010). Metodología de la Investigación (5ª ed). México: McGraw Hill.

Herrera, J. (2009). Normativa y paisaje urbano en barrios tradicionales de ciudades de cuadrícula. Montevideo: Facultad de Arquitectura.

Instituto Cervantes y Ministerio de Educación y Cultura (2002). Marco de referencia europeo para las lenguas. Aprendizaje, enseñanza y evaluación. Madrid: Artes Gráficas Fernández. 
Juliao, C. (2002). La praxeología: una teoría de la práctica. Bogotá: Uniminuto.

Juliao, C. (2011). El enfoque praxeológico. Bogotá: Uniminuto.

Juliao, C. (2014). Para entendernos en praxeología hay que re-definir la pedagogía. Bogotá: Uniminuto.

Lynch, K. (1984). La imagen de la ciudad. Barcelona: Editorial Gustavo Gili.

Ministerio de Educación Nacional (2006a). Plan nacional de educación. Bogotá D.C.: Revolución Educativa.

Ministerio de Educación Nacional (2006b). Lineamientos logros curriculares escolares. Bogotá D.C.: Revolución Educativa.

Ministerio de Educación Nacional (2006c). Lineamientos logros curriculares escolares: constitución y democracia. Bogotá D.C.: Revolución Educativa.

Ministerio de Educación Nacional (2006d). Lineamientos logros curriculares escolares: ética y valores. Bogotá D.C.: Revolución Educativa.

Ministerio de Educación Nacional (2006e). Lineamientos logros curriculares escolares: idiomas extranjeros. Bogotá D.C.: Revolución Educativa.

Ministerio de Educación Nacional (2006f). Estándares básicos de competencias ciudadanas. Bogotá D.C.: Revolución Educativa.

Ministerio de Educación Nacional (2006g). Guía 22: Estándares básicos de competencias en lenguas extranjeras: inglés. Bogotá D.C: Revolución Educativa.

Ministerio de Educación Nacional (2012). Comisión intersectorial para la atención a la primera infancia "de cero a siempre". Bogotá D.C.: Rey Naranjo Editores.

Ministerio de Educación Nacional (2014a). Documento 20. El sentido de la educación inicial. Bogotá D.C.: Rey Naranjo Editores.

Ministerio de Educación Nacional (2014b). Documento 21. El sentido de la educación inicial: el arte. Bogotá D.C.: Rey Naranjo Editores.

Ministerio de Educación Nacional (2014C). Documento 22. El sentido de la educación inicial: el juego. Bogotá D.C.: Rey Naranjo Editores.

Ministerio de Educación Nacional (2014d). Documento 23. El sentido de la educación inicial: la literatura. Bogotá D.C.: Rey Naranjo Editores.

Ministerio de Educación Nacional (2014e). Documento 24. El sentido de la educación inicial: la exploración. Bogotá D.C.: Rey Naranjo Editores. 
ISSN 0123-1294 | e-ISSN 2027-5358 | Educ.Educ. Vol. 19. No. 3 | Septiembre-Diciembre de 2016 | pp. 416-436.

Universidad de La Sabana | Facultad de Educación

Ministerio de Educación Nacional (2014f). Guía 50. Modalidad y condiciones de calidad para la educación inicial. Bogotá D.C. Rey Naranjo Editores.

Muñoz, J., Quintero, J. y Munévar, R. (2002). Experiencia en investigación acción y reflexión. Revista Electrónica de Educación Investigativa, 4 (1), 1-15.

Pérez, G. (2015). Elaboración de proyectos sociales. Madrid: Narcea.

Reyes, Y. (2005). Cuando leer es mucho más que hacer tareas. En Nuevas hojas de lectura. Bogotá D.C.: Fundalectura.

Sarramona, J. (1991). Evaluación de programas de educación a distancia. Barcelona: Anced.

Tobón, S. (2005). Aspectos básicos de la formación basada en competencias: pensamiento complejo, diseño curriculary didáctica. Bogotá D.C: Ecoe.

Tonucci, F. (1997). La ciudad de los niños. Barcelona: Grao. 